ECCOMAS

Proceedia
COMPDYN 2021

$8^{\text {th }}$ ECCOMAS Thematic Conference on Computational Methods in Structural Dynamics and Earthquake Engineering M. Papadrakakis, M. Fragiadakis (eds.) Streamed from Athens, Greece, 28 - 30 June 2021

\title{
ENERGY HARVESTING FROM RAILWAY VIBRATIONS - A NUMERICAL STUDY BASED ON BEAM-ON-ELASTIC-FOUNDATION UNDER QUASI-STATIC LOADING
}

\author{
Shahd Elshafei ${ }^{1}$, Mohammed Hussein ${ }^{1}$, Jamil Renno $^{2}$, and Asan Muthalif ${ }^{2}$ \\ ${ }^{1}$ Department of Civil \& Architectural Engineering \\ Qatar University, Doha 2713, Qatar \\ e-mail: mhussein@qu.edu.qa \\ 2 Department of Mechanical \& Industrial Engineering \\ Qatar University, Doha 2713, Qatar \\ e-mail: $\{$ jamil.renno,drasan $\} @$ qu.edu.qa
}

\begin{abstract}
The objective of this paper is to investigate energy harvesting of train-induced rail vibrations and to provide a comparison between various feasible harvesting locations based on the expected electrical power output. The vibrations of the railway track are modelled using the beam-on-elastic-foundation model under quasi-static loading. The loading applied is from a passenger train moving at a constant velocity. The vibrations of the railway track are then used to determine the energy harvested at each location using one-degree-of-freedom models. The numerical study concludes that the optimal configuration of the harvester is on the rail for the vibration harvester and as a harvesting rail pad between the sleeper and the rail for the compression harvester. The harvested energy with each configurations is $\mathcal{O}\left(\sim 10^{-6}\right)$ and $\mathcal{O}(1)$ $\mathrm{J} / \mathrm{kg}$ of harvester, respectively.
\end{abstract}

Keywords: energy harvesting, vibration energy harvester, stack harvester, ballasted track, passenger trains 


\section{Introduction}

The use of energy harvesting systems has been explored in a wide array of systems ranging from biomedical application to condition monitoring systems. Zurbuchen et al. [1] developed a customized pacemaker which was tested to harvest energy from heartbeats while pacing the heart. Research in embedding piezoelectric harvesters into knee implants showed potential in improving patient experience and enhancing motion post-surgery [2]. Yip et al. [3] presented a fully-implantable cochlea for acoustic sensing of the middle ear. Akkaya and Özdemir [4] designed and tested a cost-effective pieoelectric energy harvester that converts wind energy into useful electric energy. Mekid et al. [5] even investigated the worthiness of energy harvesting from ambient radio frequency.

To optimize the operational life of a helicopter blade, Jong et al. [6] recommended a compression (stack-type) harvester designed to monitor blade health and transmit information to the aircraft using mechanical energy. A compression harvesting system under pavements was developed by Jiang et al. [7] to investigate the feasibility of powering transport infrastructure using traffic-induced vibrations. Zhang et al. [8] conducted comprehensive simulations of various bridge properties under different loading conditions and analysed their impact on vibration-based energy harvesting. Recently, Bendine et al. [9] proposed using a vibration energy harvester in the form of a cantilever beam to harvest vibration energy from a bridge subjected to time-dependent moving loads.

This paper targets promoting energy harvesters by proposing five suitable energy harvesting configurations using vibration and stack harvesting technologies. In this paper, energy harvesting from five locations on a ballasted track using two different harvesters is investigated. The two harvesters are: vibration harvesters and compression harvesters. The vibration harvester is studied at two locations: (a) attached to the rail and (b) attached to the sleeper. The compression harvester is studied at three locations: (a) between the rail and rail pad, (b) between the rail pad and sleeper and (c) as a rail pad.

The remainder of this paper is organized as follows. In Section 2, a numerical model for the vibration of the train loading, the vibration of the track and the dynamics of the energy harvesters are presented. The models adopted for describing rail vibration and energy harvesting are described in section 2 and section 3, respectively. Section 4 presents the simulation results and analyses the energy harvesting capability of each of the five configurations for various train speeds. Conclusions for this work are drawn in section 5.

\section{Rail Vibration and Energy Harvesting}

In this section, a model for the vibration of a railway track is presented. Then, approaches to harvest energy from rail vibration will be briefly reviewed.

\subsection{Railway Track Model}

The loading applied on the rail is determined based on a typical passenger train. This choice is arbitrary and is only used for the purpose of advocating the adoption of energy harvesting capabilities in the railway sector. The developed model allows for adjustments that can later be made to accommodate for any train type. For simplification, this study considers a train made of four passenger cars of equal geometry and weight.

To calculate the loading applied by each passenger car the following is assumed:

1. The maximum seating and standing capacity is 376 passengers and thus the total weight observed accounts for the difference in the tare weight of each car type. 
2. The weight of the train is equally distributed between the cars and the weight of each car is equally distributed between eight wheels.

3. The load is transmitted to the rail as vertical point loads.

Four train speeds will be considered in the numerical simulations: 20, 40, 80 and $160 \mathrm{~km} / \mathrm{h}$. The mathematical model used to establish the train-induced vibrations of a ballasted track is the Winkler mathematical model. In order to conform to this model, the two rails in a typical track system are modelled as a single continuously-supported infinite elastic beam. The loading applied by two parallel wheels on the rail is combined to produce a single quasi-static, vertical load. Superposition is enforced to take into account the influence of successive wheels on an arbitrary location of the track, i.e., the harvester's location $z$ as shown in Figure 1.

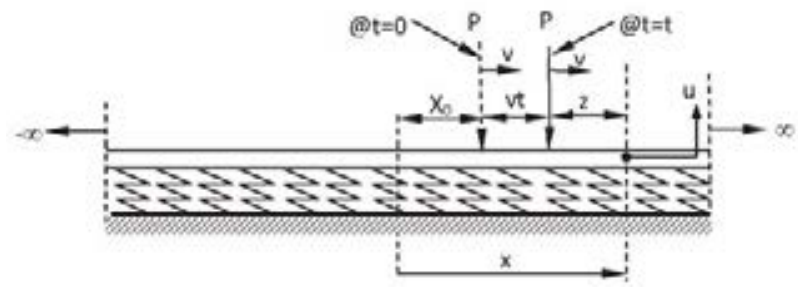

Figure 1: A beam on Elastic Foundation (Winkler model) subjected to a point load.

The vertical rail displacement $u_{r}$ at any location $z$ is given by:

$$
u_{r}(t)=-\frac{P}{8 E I \lambda^{3}} e^{-\lambda|z|}[\cos (\lambda|z|)+\sin (\lambda|z|)] \quad \text { with } \quad \lambda=\sqrt[4]{\frac{k}{4 E I}}
$$

where $E I$ is the bending stiffness of the beam representing the two rails and $k$ is the stiffness of foundation per unit length.

For a load $P$ moving with a constant velocity $v$ that is lower than the critical wave velocity of the track (i.e., quasi-static), and given that the load is at position $x_{0}$ at time $t=0$ (see Figure 1), the response of the beam at point $x$ and time $t$ can be calculated by substituting $z=x-x_{0}-v t$ in Equation 1.

The direct differentiation of Equation 1, the velocity and acceleration of the rail are numerically evaluated in preparation for the dynamic analysis in Section 3. The velocity and acceleration of the track can be obtained as:

$$
\begin{gathered}
v_{r}(t)=\left\{\begin{array}{cl}
-\frac{P v}{4 E I \lambda^{2}} e^{-\lambda z}[\sin (\lambda z)] & \forall z \geq 0 \\
-\frac{P v}{4 E I \lambda^{2}} e^{\lambda z}[\sin (\lambda z)] & \forall z \leq 0
\end{array}\right. \\
a_{r}(t)=\left\{\begin{array}{cl}
\frac{P v^{2}}{8 E I \lambda} e^{-\lambda z}[\cos (\lambda z)-\sin (\lambda z)] & z \geq 0 \\
\frac{P v^{2}}{8 E I \lambda} e^{\lambda z}[\cos (\lambda z)+\sin (\lambda z)] & z \leq 0
\end{array}\right.
\end{gathered}
$$

\subsection{Rail Stiffness}

Due to the variation in the components and their respective characteristics, in order to simulate the track as a whole system the stiffness of the various components were combined. In the simulated cases, the track components from top to bottom consist of the rail (denoted by 
subscript $r$ ), rail pads (denoted by subscript $r p$ ), sleepers (denoted by subscript $s$ ), ballast foundation (denoted by subscript $b$ ) and the ground (denoted by subscript $g$ ). As the rail displacement considered is at the web of the rail, the equivalent stiffness $\left(k_{e}\right)$ of the track system is determined using the stiffness of the components in series.

$$
\frac{1}{k_{e}}=\frac{1}{k_{r p}}+\frac{1}{k_{s}}+\frac{1}{k_{b}}+\frac{1}{k_{g}} \approx \frac{1}{k_{r p}}+\frac{1}{k_{b}}
$$

Since the stiffness of both the sleeper $k_{s}$ and ground $k_{g}$ are significantly larger than that of the ballast and rail pad, then

$$
\frac{1}{k_{e}} \approx \frac{1}{k_{r p}}+\frac{1}{k_{b}}
$$

In the following simulations, the following numerical values will be used: the bending stiffness $E I$ is assumed to be $2.52 \times 10^{7} \mathrm{Nm}^{2}$ and the equivalent stiffness $k_{e}$ is $1.66 \times 10^{8} \mathrm{~N} / \mathrm{m}$ as per the work of Cleante et al. [17].

\subsection{Base-Excitation Energy Harvesters}

The first systems that were developed to harvest energy from rail vibration were trackmounted [18]. Such harvesters rely on base-excitation ensuing from the track to convert the mechanical energy to electric energy. However, the base excitation of the track will depend on the exact mounting location (e.g. sleeper vs rail). With loading applied to the rails being directly transferred to the sleepers $\left(k_{s}\right)$, the following relationship can be used to represent the train-induced vibration of the sleeper as a factor of the rail vibration:

$$
u_{s}(t)=\frac{k_{s} k_{r}}{k_{s}+k_{g}} u_{r}(t) \approx 0.53 u_{r}
$$

where $u_{h}$ is the displacement of the harvester $m_{h}$. From Equation 2 and 3 and the rail-sleeper relationship prescribed above, it is inferred that the sleeper velocity and acceleration can be determined as a fraction of the rail velocity and acceleration, respectively as

$$
v_{s}(t)=\frac{d}{d t}\left[\frac{k_{s} k_{r}}{k_{s}+k_{g}} u_{r}(t)\right] \approx 0.53 v_{r} \quad \text { and } \quad a_{s}(t)=\frac{d^{2}}{d^{2} t}\left[\frac{k_{s} k_{r}}{k_{s}+k_{g}} u_{r}(t)\right] \approx 0.53 a_{r}
$$

where $v_{r}(t)$ and $a_{r}(t)$ were obtained in Equations 2 and 3.

Previous literature utilized vibrations at the sleeper to produce energy rather than the rail due to ease of access. This energy harvesting configuration generated an optimal electrical output in the order of $\mathrm{mW}$ which is sufficient for low-power applications such as warning signals, switches and health monitoring sensors [19]. The linear power equation adopted in many energy harvesting works:

$$
P_{h}=c_{h} v_{h}^{2}
$$

where subscript $h$ denotes the harvester, $P$ is the electrical energy harvested, $c$ is the damping and $v$ is the velocity induced by the mechanical vibrations of the rail.

\subsection{Compression-Based Energy Harvester}

Compression-based harvesters (also known as stack harvesters) are composed of two surface electrodes with opposing polarities inducing a voltage in the piezoelectric material sandwiched between them $[20,21]$. Throughout this section it is assumed that the strains are uni-directional and in the direction of the applied loading only. 


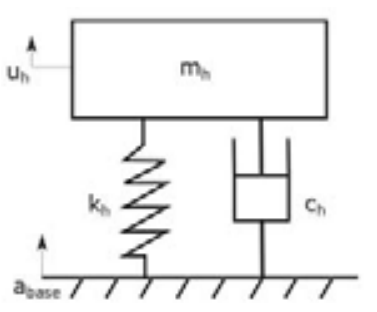

(a)

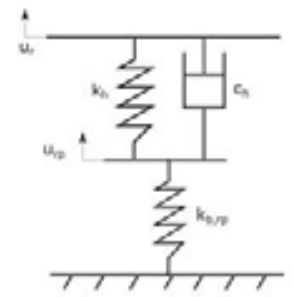

(b)

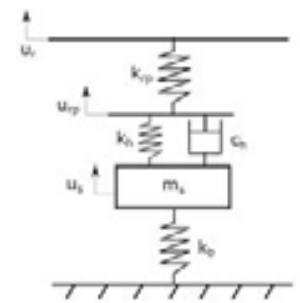

(c)

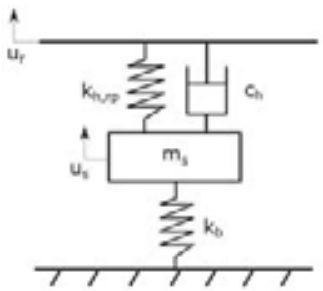

(d)

Figure 2: (a) SDOF harvester model mounted on rail (Configuration 1) or on sleeper (Configuration 2), (b) Stack harvester between the rail pad and the rail - Configuration 3, (c) Stack harvester between the rail pad and the sleeper - Configuration 4, (d) Stack harvester is used as rail pad - Configuration 5.

\section{Harvester Models}

In $[10,21]$, track-mounted harvesters were represented as single-degree-of-freedom (SDOF) systems. This methodology will be adopted here to study the performance of vibration energy harvesters and compression energy harvesters.

\subsection{Vibration Energy Harvesters}

The model shown in Figure 2a is adopted herein in two configurations: (a) the SDOF harvester is mounted on the rail (Configuration 1) or (b) mounted on the sleeper (Configuration 2).

These models can be used to determine the optimal electrical power output at each train speed. It must be noted that the dynamic model applied to both configurations is identical, the only difference is the base excitation $a_{b a s e}$ applied to the system is dependent on the mounting location of the harvester. The governing equation of the harvester in this case is

$$
m_{h} a_{h}+c_{h} v_{h}+k_{h} u_{h}=m_{h} a_{b a s e}
$$

where $m$ is mass in $\mathrm{kg}, a$ is acceleration in $\mathrm{m} / \mathrm{s}^{2}, c$ is the damping constant, $k$ is the stiffness in $\mathrm{N} / \mathrm{m}, v$ is the velocity in $\mathrm{m} / \mathrm{s}, u$ is the displacement and $a_{b a s e}$ is the base acceleration in $\mathrm{m} / \mathrm{s}^{2}$. In the forthcoming simulations, the following values will be used: $m_{h}=1, k_{h}=1.5$ and three values of the damping ratio will be used $\zeta_{h}=c_{h} / \sqrt{k_{h} m_{h}}=10^{-3}, 5.5 \times 10^{-} 3$ and $10^{-} 2$. The appropriate base acceleration $a_{\text {base }}$ is obtained from the Winkler model.

\subsection{Compression Energy Harvesters}

The compression harvester has three configurations: (a) the harvester is placed between the rail and and the rail pad (Configuration 3 in Figure 2b), (b) the harvester is placed between the rail pad and the sleeper (Configuration 4 in Figure 2c) and (c) the rail pad itself is a compression harvester (Configuration 5 in Figure 2d). The mass of the compression harvester is assumed to be only $0.004 \%$ of the sleeper's mass; therefore, the harvester is presented as a point mass for the three aforementioned configurations. The governing equations of these three configurations are presented below:

The governing equations of Configuration 3 (Figure 2b) is

$$
c_{h}\left(v_{r}-v_{r p}\right)+k_{h}\left(u_{r}-u_{r p}\right)-k_{b, r p} u_{r}=0 \quad \text { with } \quad k_{b, r p}=\frac{k_{b}}{1+\frac{k_{b}}{k_{r p}}}
$$


Table 1: Modelled compression harvester parameters

\begin{tabular}{|c|c|c|c|}
\hline Component & Mass, $m$ [kg] & Damping values $\zeta$ [dimensionless] & Stiffness $k$ [N/m] \\
\hline Rail pad, $r p$ & 0 & 0 & $2.1 \times 10^{8}$ \\
\hline Sleeper, $s$ & 192 & 0 & $\infty$ \\
\hline Harvester, $h$ & $7.5 \times 10^{-3}$ & $10^{-3}, 5.5 \times 10^{-3}, 10^{-2}$ & $7.8 \times 10^{8}$ \\
\hline
\end{tabular}

Configuration 4 (Figure 2c) is governed by the following equation

$$
m_{s} a_{s}-c_{h}\left(v_{r p}-v_{s}\right)-k_{h}\left(u_{r p}-u_{s}\right)+k_{b} u_{s}=0
$$

The governing equation of Configuration 5 (Figure 2d) is

$$
m_{s} a_{s}-c_{h}\left(v_{r}-v_{s}\right)-k_{h, r p}\left(u_{r}-u_{s}\right)+k_{b} b u_{s}=0 \quad \text { with } \quad k_{h, r p}=\frac{k_{r p}}{1+\frac{k_{h}}{k_{r p}}}
$$

where $k_{h, r p}$ is equivalent stiffness of the the harvester and rail pad.

Numerical values for the parameters of Equation 8 through Equation 10 are provided in Table 1 .

The stiffness of the rail pad and the sleeper are extracted from [20]. The sleepers are introduced into the model also undamped with negligible stiffness. As for the compression harvester, a cuboid structure of dimensions $10^{-2} \times 10^{-2} \times 10^{-2} \mathrm{~m}^{3}$ was adopted. The modelled stack harvester is purely made of piezoceramic material with a density of $7500 \mathrm{~kg} / \mathrm{m}^{3}$. Based on an averaged Young's modulus value for piezoceramic material and the set dimensions mentioned earlier, the axial stiffness of the harvester was obtained as listed in Table 1.

\section{Results and Discussion}

The simulation was run for all velocities under consideration ( $v=20,40,80,160 \mathrm{~km} / \mathrm{h}$ ). Figure $3 \mathrm{a}$ and $3 \mathrm{~b}$ shows the response of the rail under a moving passenger train at speeds 20, 40, 80 and $160 \mathrm{~km} / \mathrm{h}$. It can be noted that the sleeper displacement is $53 \%$ of the rail displacement as a result of the relationship employed in Section 2.2.

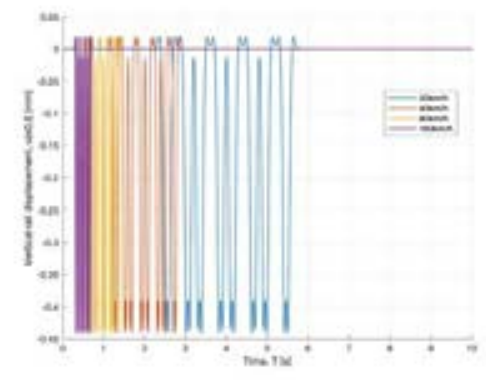

(a) Rail displacement, $u_{r}(t)$.

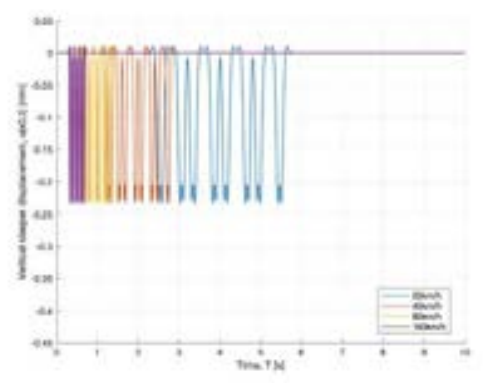

(b) Sleeper displacement, $u_{s}(t)$.

Figure 3: Vertical displacement for various train speeds, $v$, using the Winkler model.

Determining the vibration harvester velocity required the numerical integration of Equation 2a using simultaneously state representation of the $2^{\text {nd }}$ order ODE. It can be observed from Figure 5a between 8 and $20 \mathrm{~s}$ that the vibration harvester is in a transient state. The transient 
state in Figure $5 \mathrm{~b}$ is between 1 and $3 \mathrm{~s}$, far shorter and much sooner than Figure $5 \mathrm{a}$. It is evident from Figures $4 \mathrm{a}-4 \mathrm{~d}$ that with increased harvester damping, the time it takes for the harvesting system to reach rest decreases. It must be noted from Figures $4 \mathrm{a}-4 \mathrm{~d}$ that despite the train having passed, the harvester continues to oscillate and hence producing electrical output at both velocities for $t \rightarrow \infty$.

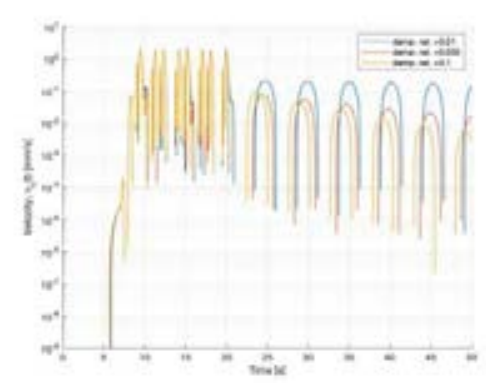

(a) Configuration $1, v=20 \mathrm{~km} / \mathrm{h}$.

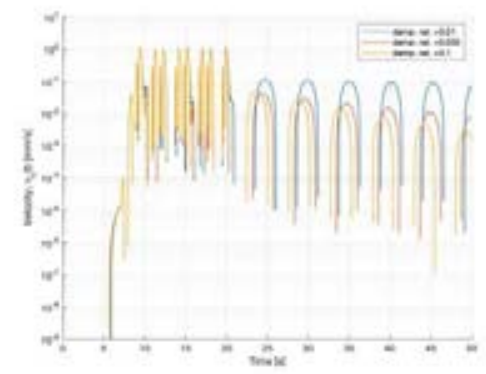

(c) Configuration 2, $v=20 \mathrm{~km} / \mathrm{h}$.

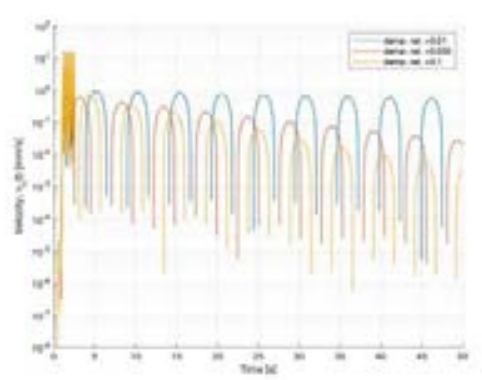

(b) Configuration 1, $v=160 \mathrm{~km} / \mathrm{h}$.

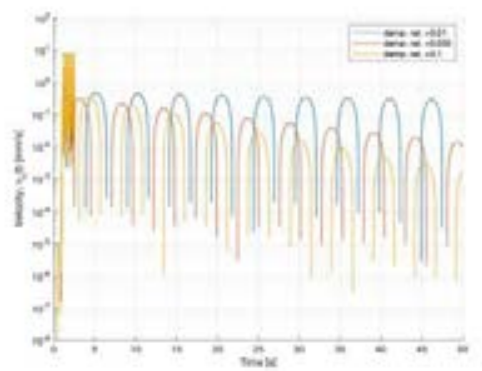

(d) Configuration $1, v=160 \mathrm{~km} / \mathrm{h}$.

Figure 4: Vertical harvester velocity, $v_{h}(t)$, for different configurations and train speeds.

From Figures 5a-5d it is observed that the peaks of power generated by the harvester correspond with the peaks in velocity from Figures $4 \mathrm{a}-4 \mathrm{~d}$. Thus, the majority of the electrical energy provided by the harvester is extracted during the transient-state. For forcing frequencies not equal to the harvester natural frequency, a larger damping is beneficial. However, greater damping of the system does not necessarily guarantee optimal power generation. This is attributed to larger damping having a detrimental affect on the oscillations of the harvester when not under resonance [14]. Table 2 presents the results of the the five investigated configurations.

Table 2: Energy harvested $E$ in $\mathrm{J} / \mathrm{kg}$

\begin{tabular}{|c|c|c|c|c|c|}
\hline & & \multicolumn{4}{|c|}{ Train speed $v$ in $\mathrm{km} / \mathrm{h}$} \\
\hline Configuration & Optimal Damping, $\zeta_{h}$ & 20 & 40 & 80 & 160 \\
\hline 1 & $10^{-2}$ & $2.85 \times 10^{-6}$ & $5.15 \times 10^{-6}$ & $9.92 \times 10^{-6}$ & $2.00 \times 10^{-5}$ \\
\hline 2 & $10^{-2}$ & $7.90 \times 10^{-7}$ & $1.43 \times 10^{-8}$ & $2.75 \times 10^{-6}$ & $5.53 \times 10^{-6}$ \\
\hline 3 & $10^{-3}$ & $1.45 \times 10^{6}$ & $3.82 \times 10^{5}$ & $1.84 \times 10^{5}$ & $9.13 \times 10^{4}$ \\
\hline 4 & $10^{-3}$ & $5.38 \times 10^{4}$ & $1.45 \times 10^{2}$ & $3.00 \times 10^{1}$ & $3.93 \times 10^{10}$ \\
\hline 5 & $10^{-2}$ & $7.18 \times 10^{-2}$ & $1.36 \times 10^{-1}$ & $2.71 \times 10^{-1}$ & 5.19 \\
\hline
\end{tabular}




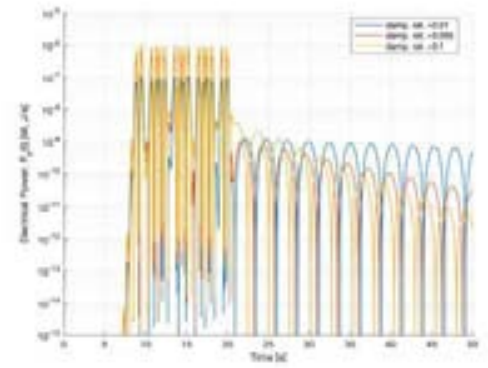

(a) Configuration $1, v=20 \mathrm{~km} / \mathrm{h}$.

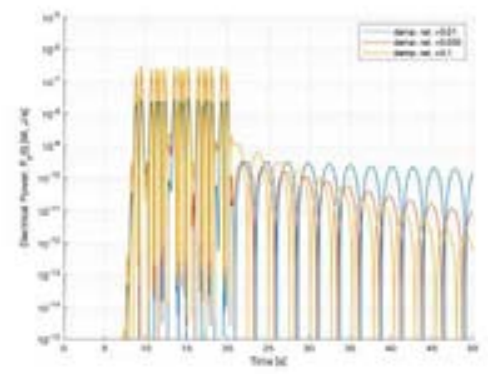

(c) Configuration 2, $v=20 \mathrm{~km} / \mathrm{h}$.

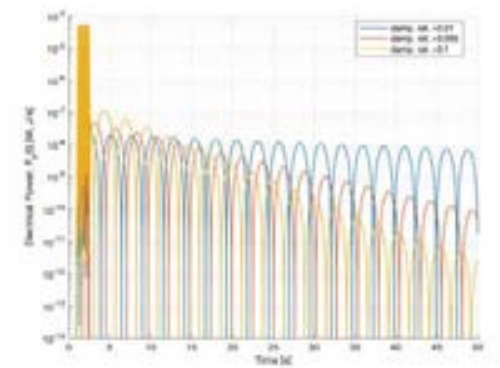

(b) Configuration $1, v=160 \mathrm{~km} / \mathrm{h}$.

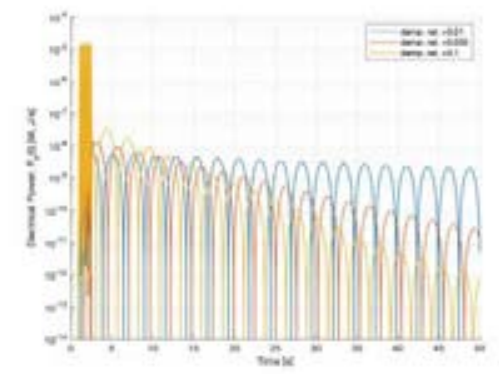

(d) Configuration 2, $v=160 \mathrm{~km} / \mathrm{h}$.

Figure 5: Harvested electric power, $P_{h}(t)$, for different harvester configuration and at different train velocities.

\section{Conclusions}

To articulate the potential of harvester technology in providing reliable, safe and green energy to power and monitor appliances in industry, this paper proceeded in utilizing preliminary dynamic models and a typical passenger train to propose an optimal harvesting configuration for railway application. Current literature on railway harvesting are limited to vibration energy harvesting from sleepers due to ease of access and installation in comparison to alternative locations. This paper aspires to numerically model multiple harvesting configurations using MATLAB and determining an optimal harvesting location and corresponding harvester properties based on performance. Firstly the beam-on-elastic-foundation model for a continuouslysupported infinite beam on ballast foundation was employed to determine the rail responses under train-induced quasi-static loading. The train modelled was a typical passenger train. Simplified mass-spring damper models were used to represent five different configurations of energy harvesters. The five configurations considered are labelled 1-5 are in the following order: Electromagnetic harvester placed on the rails, Electromagnetic harvester placed on the sleeper, Stack of harvesters above the rail pads, Stack of harvesters between rail pads and sleepers, Stack harvesting rail pad. The energy harvested at each configuration for a number of train speeds was evaluated. It was concluded that Configuration 1 is the most favourable for vibration harvester for all train speeds. As for the stack harvester, the more advantageous configuration is a variable of the train speed. Overall, Configuration 3 and 4 are better at generating electrical energy under the same conditions than Configuration 5. 


\section{Acknowledgment}

This paper has been carried out under a research project entitled "Framework for Research on Railway Engineering" which is supported by a grant sponsored by Qatar Rail and managed by Qatar University under reference number: QUEX-CENG-Rail 17/18.

\section{REFERENCES}

[1] A. Zurbuchen, A. Haeberlin, L. Bereuter, J. Wagner, A. Pfenniger, S. Omari, J. Schaerer, F. Jutzi, C. Huber, J. Fuhrer, and R. Vogel. The swiss approach for a heartbeat-driven leadand batteryless pacemaker. Heart Rhythm, 14(2):294-299, 2017.

[2] M. Safaei, R.M. Meneghini, and S.R. Anton. Energy harvesting and sensing with embedded piezoelectric ceramics in knee implants. IEEE/ASME Transactions on Mechatronics, 23(2):864-874, 2018.

[3] M. Yip, R. Jin, H.H. Nakajima, K.M. Stankovic, and A.P. Chandrakasan. A fullyimplantable cochlear implant soc with piezoelectric middle-ear sensor and arbitrary waveform neural stimulation. IEEE Journal of Solid-State Circuits, 50(1):214-229, 2015.

[4] S. Akkaya Oy and A.E. Özdemir. Piezoelectric-based low-power wind generator design and testing. Arabian Journal for Science and Engineering, 43(6):2759-2767, 2018.

[5] S. Mekid, A. Qureshi, and U. Baroudi. Energy harvesting from ambient radio frequency: Is it worth it? Arabian Journal for Science and Engineering, 42(7):2673-2683, 2017.

[6] P.H. De Jong, A. De Boer, R. Loendersloot, and P.J.M. Van Der Hoogt. Power harvesting in a helicopter rotor using a piezo stack in the lag damper. Journal of Intelligent Material Systems and Structures, 24(11):1392-1404, 2013.

[7] X. Jiang, Y. Li, J. Li, J. Wang, and J. Yao. Piezoelectric energy harvesting from trafficinduced pavement vibrations. Journal of Renewable and Sustainable Energy, 6(4), 2014.

[8] Y. Zhang, S.C.S. Cai, and L. Deng. Piezoelectric-based energy harvesting in bridge systems. Journal of Intelligent Material Systems and Structures, 25(12):1414-1428, 2014.

[9] K. Bendine, M. Hamdaoui, and B.F. Boukhoulda. Piezoelectric energy harvesting from a bridge subjected to time-dependent moving loads using finite elements. Arabian Journal for Science and Engineering, 44(6):5743-5763, 2019.

[10] T. Lin, J.J. Wang, and L. Zuo. Efficient electromagnetic energy harvester for railroad transportation. Mechatronics, 53:277-286, 2018.

[11] M. Gao, P. Wang, Y. Cao, R. Chen, and D. Cai. Design and verification of a rail-borne energy harvester for powering wireless sensor networks in the railway industry. IEEE Transactions on Intelligent Transportation Systems, 18(6):1596-1609, 2017.

[12] V.G. Cleante, M.J. Brennan, G. Gatti, and D.J. Thompson. On the target frequency for harvesting energy from track vibrations due to passing trains. Mechanical Systems and Signal Processing, 114:212-223, 2019. 
[13] J. Ortiz, P.M. Monje, N. Zabala, M. Arsuaga, J. Etxaniz, and G. Aranguren. New proposal for bogie-mounted sensors using energy harvesting and wireless communications. Proceedings of the Institution of Mechanical Engineers, Part F: Journal of Rail and Rapid Transit, 228(7):807-820, 2014.

[14] G. Gatti, M.J. Brennan, M.G. Tehrani, and D.J. Thompson. Harvesting energy from the vibration of a passing train using a single-degree-of-freedom oscillator. Mechanical Systems and Signal Processing, 66-67:785-792, 2016.

[15] C.A. Nelson, A. Pourghodrat, and M. Fateh. Energy harvesting from vertical deflection of railroad track using a hydraulic system for improving railroad track safety. In ASME 2011 International Mechanical Engineering Congress and Exposition, IMECE 2011, volume 3, pages 259-266, 2011.

[16] J. Wang, Z. Shi, H. Xiang, and G. Song. Modeling on energy harvesting from a railway system using piezoelectric transducers. Smart Materials and Structures, 24(10), 2015.

[17] V.G. Cleante, M.J. Brennan, G. Gatti, and D.J. Thompson. Energy harvesting from the vibrations of a passing train: Effect of speed variability. In Journal of Physics: Conference Series, volume 744, 2016.

[18] C.A. Nelson, S.R. Platt, S.E. Hansena, and M. Fateh. Power harvesting for railroad track safety enhancement using vertical track displacement. In Proceedings of SPIE - The International Society for Optical Engineering, volume 7288, 2009.

[19] J.J. Wang, G.P. Penamalli, and L. Zuo. Electromagnetic energy harvesting from train induced railway track vibrations. In Proceedings of 2012 8th IEEE/ASME International Conference on Mechatronic and Embedded Systems and Applications, MESA 2012, pages 29-34, 2012.

[20] B. C. Kok, Saleh Gareh, H. H. Goh, and C. Uttraphan. Electromechanical-traffic model of compression-based piezoelectric energy harvesting. In International Conference on Manufacturing and Industrial Technologies, volume 70, 2016.

[21] D.R.M. Milne, L.M. Le Pen, D.J. Thompson, and W. Powrie. Properties of train load frequencies and their applications. Journal of Sound and Vibration, 397:123-140, 2017. 